\title{
INTERPOLATION TO ANALYTIC DATA ON UNBOUNDED CURVES
}

\author{
BY \\ MAYNARD THOMPSON $\left({ }^{1}\right)$
}

\begin{abstract}
This paper provides a method for constructing a family of sets of points on the boundary (assumed suitably smooth) of an unbounded Jordan region in the complex plane which is useful for certain interpolation problems. It is proved that if these sets are used as nodes for Lagrange interpolation to analytic data, then the resulting polynomials converge in the region, and the limit function is related in a natural way to the boundary data. Subsidiary results include an approximate quadrature formula for slowly decreasing functions on an infinite interval.
\end{abstract}

1. Introduction. There is a well-known theorem due to L. Fejér [3] which asserts that if $\Omega$ is a bounded region in the complex $z$-plane whose boundary $\Gamma$ is a Jordan curve, then one can find a family $\left\{S_{n}\right\}$ of sets of points on $\Gamma$, each $S_{n}$ containing $n$ distinct points, with the following property. If $f$ is any function analytic on $\Omega \cup \Gamma$, then the sequence $\left\{L_{n}[f ; \cdot]\right\}$ of polynomials of respective degrees $n-1$ which interpolate to $f$ on $S_{n}$ satisfies

$$
\lim _{n \rightarrow \infty} L_{n}[f ; z]=f(z)
$$

uniformly for $z \in \Omega \cup \Gamma$. Fejér's result has been extended and refined by J. L. Walsh ([5], [6, Chapter V] and [7, Chapter VII]), but attention has been restricted to bounded regions $\Omega$. The purpose of this paper is to present an analogous theorem (Theorem 1) which holds for certain unbounded regions $\Omega$. Briefly, the conditions on $\Omega$ imposed here relate to the size of $\Omega$ at $\infty$ and the behavior of the boundary $\Gamma$ of $\Omega$.

A detailed study of the admissible regions is given in $\$ 2$, and the proof of the main theorem is developed in several steps in $\$ \S 3-5$. The final section is devoted to comments on the admissibility conditions and another interpretation of the main theorem.

The author wishes to acknowledge helpful discussions with Professors J. Korevaar, especially regarding Lemma 2 , and J. L. Walsh.

2. Admissible regions. Let $\Omega$ be an unbounded Jordan region in the complex

Received by the editors March 12, 1971.

AMS 1970 subject classifications. Primary 30A80; Secondary 30A82, 41A55.

Key words and phrases. Polynomial interpolation on unbounded regions, rational approximation, approximate quadrature.

$\left({ }^{1}\right)$ This work supported in part by National Science Foundation grant GP 20044.

Copyright (C) 1972, American Mathematical Society 
$z$-plane with boundary $\Gamma$, or equivalently, a Jordan region on the sphere whose boundary contains the north pole. For technical reasons it is convenient to assume that the origin of the $z$-plane is contained in $\Omega$. We will consider here only those regions whose boundary has half tangents at $\infty$. By this we mean that the curve $\Gamma^{\prime}$ in the $\mathscr{Z}$-plane whose trace is $\{\mathscr{Z}: \mathscr{Z}=1 / z, z \in \Gamma\} \cup\{0\}$ has half tangents at $\mathscr{Z}=0$. If the angle subtended in the $\mathscr{Z}$-plane by the set $\Omega^{\prime}=\{\mathscr{Z}: \mathscr{Z}=1 / z, z \in \Omega\}$ at $\mathscr{Z}=0$ is $\beta \pi$, then we say that $\Omega$ has opening $\beta \pi$ at $\infty$. Next, let $z_{1} \in \Gamma$ be such that $\left|z_{1}\right|=\min \{|z|: z \in \Gamma\}$ and for each $R>\left|z_{1}\right|$ define an arc $\gamma_{R}$ as follows. Beginning at $z_{1}$ traverse the curve $\Gamma$ in one direction from $z_{1}$, intersecting the circle $|z|=R$ from time to time, until the last such point of intersection is reached and call this point $P$. Proceeding on $\Gamma$ in the other direction from $z_{1}$, let the last point of intersection of $\Gamma$ with $|z|=R$ be denoted $Q$. The $\operatorname{arc} \gamma_{R}$ is the subarc of $\Gamma$ which contains $z_{1}$ and whose endpoints are $P$ and $Q$. Define $\Delta(R)=\sup \left\{|z|: z \in \gamma_{R}\right\}$. If

$$
\limsup _{R \rightarrow \infty} \frac{\Delta(R)}{R}<\infty,
$$

then we say that $\Gamma$ satisfies condition $\mathrm{W}$ at $\infty$. The function $\Delta(R)$ is the "Unbewalltheitsfunktion" of $S$. Warschawski for the curve $\Gamma$ and the point $\infty$ ([8], [9]). We remark that (1) is satisfied, for example, if $|z|$ is eventually a nondecreasing function as $z$ tends to $\infty$ in both directions on $\Gamma$.

With $z_{1}$ as above let $\Phi$ be any (fixed) one-to-one conformal mapping of the upper half of the $w$-plane, $\operatorname{Im} w>0$, onto the complement $\Omega^{*}$ of $\Omega \cup \Gamma$ which satisfies $\Phi(\infty)=\infty, \Phi(0)=z_{1}$. The function $\Phi$ can be extended to a homeomorphism of $\operatorname{Im} w \geqq 0$ onto $\Omega^{*} \cup \Gamma$, and its restriction $\phi$ to $\operatorname{Im} w=0$ provides a parametric representation of the boundary $\Gamma$ of $\Omega^{*}($ or $\Omega$ )

$$
\Gamma: t \rightarrow \phi(t), \quad-\infty<t<\infty .
$$

Note that the orientation of $\Gamma$ induced by this parametrization is positive with respect to $\Omega^{*}$.

The following lemma is a slightly restated version of Theorem 6 of [8]. The original involves conformal mappings of bounded regions onto the disc. The auxiliary mappings necessary to obtain the form given here are obvious.

LEMMA 1. Let $\Omega$ have opening $(2-\alpha) \pi, 0<\alpha<2$, at $\infty$, let its boundary $\Gamma$ satisfy condition $\mathrm{W}$, and let $\Phi$ be the conformal mapping introduced above. Then for every $\varepsilon>0$ there is a positive real number $R=R(\varepsilon)$ such that $|w|^{\alpha-\varepsilon}<|\Phi(w)|<|w|^{\alpha+\varepsilon}$ for all $w$ with $\operatorname{Im} w \geqq 0,|w| \geqq R$.

We say that $\Gamma$ is rectifiable on the sphere if

(i) every bounded subarc is rectifiable, and consequently $\phi$ is differentiable almost everywhere on $(-\infty, \infty)$, and

$$
\int_{-\infty}^{\infty} \frac{|d \phi / d t|}{|\phi(t)|^{2}} d t<\infty
$$


We remark that these conditions are equivalent to the following assumption: the bounded Jordan curve defined by $\theta \rightarrow[\phi((\sin \theta) /(\cos \theta-1))]^{-1}$ is rectifiable.

With these preliminaries we can identify the regions to be considered in this paper.

Definition. An unbounded Jordan region $\Omega$ is said to be admissible if the following conditions are satisfied:

(a) $\Omega$ has opening $A, 0<A<\pi$, at $\infty$,

(b) the boundary $\Gamma$ of $\Omega$ satisfies condition $\mathrm{W}$,

(c) the boundary $\Gamma$ of $\Omega$ is rectifiable on the sphere.

It is important for what follows to note that condition (c) also implies that $\phi$ is absolutely continuous on every closed subinterval of $(-\infty, \infty)$. We adopt standard terminology and say that $\phi$ is locally absolutely continuous on $(-\infty, \infty)$.

We observe that an appropriate translate of a sector with opening smaller than $\pi$ is an admissible region. Thus for each $A, 0<A<\pi$, the region $\Omega_{A}=\left\{z: z=1-r e^{i \theta}\right.$, $-A / 2<\theta<A / 2,0<r<\infty\}$ is admissible. A region with a smooth boundary which has much the same geometry as $\Omega_{A}$ at $\infty$ is the region containing $z=0$ whose boundary is $\Gamma_{A}$,

$$
\Gamma_{A}=\left\{z: z=\left|1+t^{2}\right|^{\alpha / 2} e^{i \alpha \operatorname{Arg}(1+i t)},-\infty<t<\infty\right\}
$$

where $\alpha=(2-A) / \pi$ and that branch of the argument is selected which is zero for $t=0$.

3. Quadrature lemmas. We adopt the convention that if $r$ is a positive real number, then $[r]$ denotes the largest integer which is not larger than $r$, i.e. $[r]=\sup \{m: m \leqq r, m$ an integer $\}$.

LEMMA 2. Let $f$ be a complex valued function defined on $(-\infty, \infty)$ satisfying

(i) $f$ is locally absolutely continuous on $(-\infty, \infty)$,

(ii) $f^{\prime} \in L_{1}(-\infty, \infty)$,

(iii) $f(t)=O\left(|t|^{-\mu}\right), \mu>1$, as $|t| \rightarrow \infty$.

Then there is a constant $\lambda>1$ such that

$$
\sum_{|k| \leqq\left[n^{\lambda}\right]} f(k / n)-n \int_{-\infty}^{\infty} f(t) d t=o(1), \quad n \rightarrow \infty .
$$

Proof. It is sufficient to prove the lemma for real valued functions $f$. Let $I(n)$ denote the expression on the left-hand side of (2). Using condition (iii) and properties of the greatest integer function $[\cdot]$ we obtain

$$
|I(n)| \leqq\left|\sum_{|k| \leqq\left[n^{\lambda}\right]} f(k / n)-n \int_{-\left[n^{\lambda}\right] / n}^{\left[n^{\lambda}\right] / n} f(t) d t\right|+n O\left(n^{(\lambda-1)(1-\mu)}\right) .
$$

Select $\lambda>\mu /(\mu-1)$. Then $(1-\mu)(\lambda-1)+1<0$ and the last term on the right-hand side of (3) is $o(1)$ as $n \rightarrow \infty$. It remains to show that the first term on the right-hand side behaves similarly. To this end, we note that the term in question can be written 


$$
\begin{aligned}
\left|f\left(-\left[n^{\lambda}\right] / n\right)+\sum_{|k| \leqq\left[n^{\lambda}\right]} n \int_{(k-1) / n}^{k / n}\left(f\left(\frac{k}{n}\right)-f(t)\right) d\left(t-\frac{k-1}{n}\right)\right| \\
=\left|n \sum_{|k| \leqq\left[n^{\lambda}\right]} \int_{(k-1) / n}^{k / n}\left(t-\frac{k-1}{n}\right) f^{\prime}(t) d t\right|+o(1) .
\end{aligned}
$$

Now, for any $\varepsilon>0$ pick an interval $I=[-a, a]$ such that $|f(t)|<\varepsilon, t \notin I$, and $\int_{-\infty}^{-a}\left|f^{\prime}\right|+\int_{a}^{\infty}\left|f^{\prime}\right|<\varepsilon$. Henceforth when an integer $n$ is mentioned it is to be understood that $n$ satisfies $\left[n^{\lambda}\right]>a$, where $\lambda$ is the constant selected above. Finally, let $Q$ be the set of all integers and define

$$
\begin{aligned}
K & =K(n)=\left\{k: k \in Q,|k| \leqq\left[n^{\lambda}\right], \text { and }[(k-1) / n, k / n] \cap I=\varnothing\right\}, \\
K^{*} & =K^{*}(n)=\left\{k: k \in Q,|k| \leqq\left[n^{\lambda}\right], k \notin K\right\} .
\end{aligned}
$$

We can now estimate the sum in (4). First, since $|n(t-(k-1) / n)| \leqq 1$ for $t \in[(k-1) / n, k / n]$, we have

$$
\left|n \sum_{k \in K} \int_{(k-1) / n}^{k / n}\left(t-\frac{k-1}{n}\right) f^{\prime}(t) d t\right| \leqq \int_{-\infty}^{-a}\left|f^{\prime}\right|+\int_{a}^{\infty}\left|f^{\prime}\right|<\varepsilon .
$$

The remaining terms in (4) can be estimated by comparing them with the integral of $f^{\prime}$ (see [1], [4])

$$
\begin{aligned}
\mid \sum_{k \in K^{*}} n \int_{(k-1) / n}^{k / n}\left(t-\frac{k-1}{n}\right) & f^{\prime}(t) d t \mid \\
& =\left|\frac{1}{2} \int_{-a}^{a} f^{\prime}(t) d t\right|+o(1)=\left|\frac{f(a)-f(-a)}{2}\right|+o(1), \quad n \rightarrow \infty .
\end{aligned}
$$

The right-hand side of this last expression can be made less than $2 \varepsilon$ by taking $n$ sufficiently large, and our proof is complete.

It is important for our work that this lemma holds with a certain uniformity if the function $f$ depends analytically on a complex parameter. If we let $G$ be a region in the complex $z$-plane the precise statement is the following.

LEMMA 3. Let $f(z, t)$ be a complex valued function defined on $G \times(-\infty, \infty)$ satisfying

(i) $f(\cdot, t)$ is analytic on $G,-\infty<t<\infty$,

(ii) $f(z, \cdot)$ is locally absolutely continuous for each fixed $z \in G$, and

$$
\int_{-\infty}^{\infty}\left|\frac{d f}{d t}(z, t)\right| d t
$$

is uniformly bounded on compact subsets of $G$,

(iii) $|f(z, t)|=O\left(|t|^{-\mu}\right), \mu>1,|t| \rightarrow \infty$, with the right-hand side uniform on compact subsets of $G$. Then there is a constant $\lambda>1$ such that

$$
\sum_{|k| \leqq\left[n^{\lambda}\right]} f(z, k / n)-n \int_{-\infty}^{\infty} f(z, t) d t=o(1), \quad n \rightarrow \infty,
$$

with the right-hand side uniform on compact subsets of $G$. 
Proof. For each fixed $z \in G$ the conclusion follows at once from Lemma 2 . Moreover, the constant $\lambda$ may be selected independent of $z$. In fact any fixed number larger than $\mu /(\mu-1)$ will serve as a satisfactory $\lambda$. To establish the uniformity we prove that the left-hand side of $(5)$, denoted $I(n, z)$, is uniformly bounded on compact subsets of $G$, and then we apply Vitali's Theorem. The proof of boundedness is direct. Indeed, an argument analogous to that of the proof of Lemma 2 can be given under the hypotheses of the present lemma. This produces an inequality similar to (4) which holds uniformly on compact subsets of $G$. The resulting inequality is

$$
|I(n, z)| \leqq \int_{-\infty}^{\infty}\left|\frac{d f}{d t}(z, t)\right| d t+O\left(n^{(1-\mu)(\lambda-1)+1}\right),
$$

where the $O$ term is uniform on compact subsets of $G$. Since $\lambda>\mu /(\mu-1)$ the second term is actually $O(1)$ uniformly on compact subsets of $G$, and the uniform boundedness on compact subsets of the first term follows from hypothesis (ii).

4. Estimates on the fundamental polynomials. The fundamental polynomials of Lagrange interpolation are usually written in the form

$$
\omega_{n}(z)=\prod_{k=1}^{n}\left(z-z_{n k}\right), \quad n=n_{1}, n_{2}, \ldots, n_{j} \rightarrow \infty,
$$

where $S_{n}=\left\{z_{n k}\right\}_{k=1}^{n}$ is the $n$th interpolating set. However, in the situation under investigation here there is no finite bound on the moduli of the points in the set $S_{n}$ as $n$ becomes large, and consequently it is convenient to consider instead the normalized polynomials

$$
p_{n}(z)=\prod_{k=1}^{n}\left(1-\frac{z}{z_{n k}}\right), \quad z_{n k} \neq 0, \quad k=1,2, \ldots, n,
$$

$n=n_{1}, n_{2}, \ldots, n_{j} \rightarrow \infty$. In the derivation of estimates on these polynomials it is frequently useful to introduce their logarithms

$$
\log p_{n}(z)=\sum_{k=1}^{n} \log \left(1-\frac{z}{z_{n k}}\right)
$$

where the meaning assigned to this equation must be made precise. Recall that our goal is to obtain theorems on the convergence of polynomials which interpolate to a given function on $\Gamma$. With this in mind, we introduce the (extended) mapping function $\Phi$ of $\S 1$ and write $z_{n k}=\Phi\left(t_{n k}\right),-\infty<t_{n k}<\infty, k=1,2, \ldots, n, n=n_{1}, n_{2}, \ldots$, $n_{j} \rightarrow \infty$. Thus (8) leads to a consideration of the function

$$
g(z, w)=\log (1-z / \Phi(w)),
$$

where $z \in \Omega$ and $\operatorname{Im} w \geqq 0$. In (9) we take that branch of the logarithm which assumes the value 0 for $z=0$. This convention naturally makes (8) well defined.

According to Lemma 1 and the definitions, if $\Omega$ is admissible, then the function $g$ 
defined on $\Omega \times(-\infty, \infty)$ satisfies the hypotheses of Lemma 3 . Thus we obtain the following, actually a result involving asymptotically neutral families [2].

LEMMA 4. If $\Omega$ is admissible, then there is a constant $\lambda$ such that

$$
\prod_{|k| \leq\left[n^{\lambda}\right]}\left(1-\frac{z}{\Phi(k / n)}\right) \rightarrow 1, \quad n \rightarrow \infty,
$$

uniformly on compact subsets of $\Omega$.

Proof. The conclusion is an immediate consequence of Lemma 3 and the fact

$$
\int_{-\infty}^{\infty} g(z, t) d t=0, \quad z \in \Omega .
$$

Equation (11) is a consequence of the Cauchy integral theorem. To see this, let $G_{R}$ denote the positively oriented contour in the $w$-plane consisting of the segment of the real axis from $-R$ to $R$ and the semicircle in the upper half plane with this as diameter. For each fixed $z \in \Omega$ the function $g(z, w)$ is an analytic function of $w$, and therefore $\int_{G_{R}} g(z, w) d w=0$, or

$$
\int_{-R}^{R} g(z, t) d t+\int_{0}^{\pi} g\left(z, \operatorname{Re}^{i \theta}\right) i R e^{i \theta} d \theta=0 .
$$

For $R$ sufficiently large the integrand $g\left(z, R e^{i \theta}\right)$ can be expanded in a power series in $z / \Phi\left(R e^{i \theta}\right)$, and if we use the estimate of Lemma 1 , then let $R$ increase to $\infty$, we have (11). Incidentally, this expansion also shows that the integral in (11) exists as an ordinary improper integral and not only in the Cauchy principal value sense.

LeMmA 5. Let $\Omega$ be admissible and $E$ a compact set contained in $\Omega^{*}$,

$$
\delta=\min \left\{\operatorname{Im} \Phi^{-1}(z), z \in E\right\} .
$$

Then there is a constant $\lambda$ such that

$$
\prod_{|k| \leqq\left[n^{\lambda}\right]}\left|1-\frac{z}{\Phi(k / n)}\right| \geqq \exp (2 \pi n \delta+O(1)), \quad z \in E, \quad n \rightarrow \infty,
$$

where the $O$ term is uniform on $E$. Moreover, $\lambda$ can be selected independent of $E$ and such that (10) and (12) are both true.

Proof. The crux of the proof is the estimate

$$
\left|\sum_{|k| \leqq\left[n^{\lambda}\right]} \log \right| 1-\frac{z}{\Phi(k / n)}\left|-2 \pi n \operatorname{Im} \Phi^{-1}(z)\right|=O(1),
$$

$z \in E, n \rightarrow \infty$, where the $O$ term is uniform on $E$. In (13) we take that branch of the logarithm defined for $z \in \Omega *$ which satisfies

$$
\lim _{t \rightarrow \infty} \log (1-z / \Phi(t))=0 .
$$

We proceed with the proof of (13). Let $G_{R}$ be the contour introduced in the proof 
of Lemma 4, and henceforth suppose $R>\max \left\{\left|\Phi^{-1}(z)\right|, z \in E\right\}$. Consider a fixed $z_{0}$ and set $w_{0}=\Phi^{-1}\left(z_{0}\right)$. Construct a contour $G(R, r, \varepsilon)$ as follows: Let $\gamma_{r}$ denote the circle with center $w_{0}$ and radius $r, 0<r<\operatorname{distance}\left(w_{0}, G_{R}\right)$, and $\sigma_{r}$ the vertical segment of length $\operatorname{Im} w_{0}-r$ from $\rho=w_{0}-i r$ to $q=\operatorname{Re} w_{0}$. For $\varepsilon<r$ we define $G(R, r, \varepsilon)$ to be the positively oriented contour in the $w$-plane consisting of that segment of the real axis from $-R$ to $q-\varepsilon$, the vertical segment from $q-\varepsilon$ to $\gamma_{r}$, that portion of $\gamma_{r}$ given by $w_{0}+r e^{-i \theta}, \pi-\beta \leqq \theta \leqq 2 \pi+\beta, \beta=\cos ^{-1}(\varepsilon / r)$, the vertical segment from $w_{0}+r e^{-i(2 \pi+\beta)}$ to $q+\varepsilon$, that segment of the real axis from $q+\varepsilon$ to $R$, and finally the semicircle in $\operatorname{Im} w \geqq 0$ with the segment $(-R, R)$ as diameter. Geometrically, the domain bounded by $G(R, r, \varepsilon)$ looks like that bounded by $G_{R}$ with a keyhole centered at $w_{0}$ removed. Return for a moment to the function $g$ defined by (9). This definition was given for $z \in \Omega$, but for any $z \in \Omega^{*}$ the same formula can be used to define an analytic function of $w$ on any simply connected subregion of $\operatorname{Im} w>0$ not containing $\Phi^{-1}(z)$. Now, with $z_{0}$ and $w_{0}$ as above, let $g\left(z_{0}, w\right)$, or equivalently $g\left(\Phi\left(w_{0}\right), w\right)$, denote the function defined by (9) in the region consisting of the upper half of the $w$-plane with the vertical segment connecting $w_{0}$ and the real axis removed. By selecting the appropriate branch of the logarithm, we can guarantee that $g\left(z_{0}, w\right)$ tends to zero as $w$ tends to $\infty$ in the upper half plane. The function $g\left(z_{0}, w\right)$ is analytic inside $G(R, r, \varepsilon)$ and continuous on the closure of this region, and therefore the integral of $g\left(z_{0}, \cdot\right)$ around this contour is zero. Letting $\varepsilon \rightarrow 0, r \rightarrow 0$ and applying standard estimates we obtain

$$
\int_{-R}^{R} \operatorname{Re} g\left(z_{0}, t\right) d t-2 \pi \operatorname{Im} w_{0}=-\operatorname{Re}\left\{\int_{0} g\left(z_{0}, R e^{i \theta}\right) i R e^{i \theta} d \theta\right\} .
$$

This equation is independent of the branch of the logarithm selected above. Next, take $R$ such that

$$
\underset{0 \leqq \theta \leqq \pi}{\operatorname{minimum}}\left|\Phi\left(R e^{i \theta}\right)\right|>\underset{z \in E}{\operatorname{maximum}}|z|
$$

and expand $g\left(z_{0}, R e^{i \theta}\right)$ in a power series in $z_{0} / \Phi\left(R e^{i \theta}\right)$. Again it follows from Lemma 1 that the right-hand side of (14) is $O\left(R^{1-\mu}\right), \mu>1$, and the $O$ is uniform for $z_{0} \in E$. Using the same techniques as in $\S 3$ we obtain

$$
\begin{aligned}
\mid \sum_{|k| \leqq\left[n^{\lambda}\right]} g(z, k / n) & -n \int_{-\left[n^{\lambda}\right]}^{\left[n^{\lambda}\right]} g(z, t) d t \mid \\
& \leqq \int_{-\infty}^{\infty}\left|\frac{d g}{d t}(z, t)\right| d t+O\left(n^{\mu(1-\lambda)}\right)=O(1), \quad n \rightarrow \infty,
\end{aligned}
$$

where the $O$ terms are uniform on $E$. This last inequality together with (14), with $R=\left[n^{\lambda}\right]$, gives (13). The estimate (12) follows from (13) by exponentiation and the definition of $\delta$.

5. Convergence theorems. The lemmas of the preceding sections provide the technical results necessary for our proof of the following theorem, the principal theorem of this paper. 
THEOREM 1. Let $\Omega$ be an admissible region with boundary $\Gamma$. Then there is a family $\left\{S_{N}\right\}$ of sets of points on $\Gamma, S_{N}=\left\{z_{N k}\right\}_{k=1}^{N}, N=n_{1}, n_{2}, \ldots, n_{j} \rightarrow \infty$, such that if $f$ is any nonconstant function analytic on $\Omega \cup \Gamma$ (including $\infty$ ), then the sequence $L_{N}[f ; \cdot]$ of polynomials of respective degrees $N-1$ which interpolate to $f$ on $S_{N}$ satisfies

$$
\lim _{N \rightarrow \infty} L_{N}[f ; z]=f(z), \quad z \in \Omega,
$$

and the convergence is uniform on compact subsets of $\Omega$.

We prove the theorem by exhibiting a family $\left\{S_{N}\right\}$ with the property that if $f$ is any such function and $F$ is a compact subset of $\Omega$, then (15) holds uniformly on $F$. Since the Lagrange interpolation operators $L_{N}: f \rightarrow L_{N}[f ; \cdot]$ are linear, it is no restriction to assume $f(\infty)=0$. We proceed with this additional assumption.

Let $\Phi$ be the (extended) mapping function of $\S 2$, and let $h>0$ be selected so that $\Omega \cup\{z: z=\Phi(w), 0 \leqq \operatorname{Im} w \leqq h\}$ is contained in the region of analyticity of $f$. Let $\Gamma_{h}$ be the unbounded analytic Jordan arc given by $\Phi(t+i h),-\infty<t<\infty$, and set $\rho_{0}=|\Phi(i h)|$. Since $f$ is analytic at $\infty$, there is a real number $\rho_{\infty}$ such that $f$ is analytic outside the disc with center 0 and radius $\rho_{\infty}$. Set $R_{0}=\max \left\{\rho_{0}, \rho_{\infty}, \sup _{z \in F}|z|\right\}$, and for each $R>R_{0}$ define two contours $G_{R h}^{1}$ and $G_{R h}^{0}$ as follows. Let

$$
\begin{aligned}
& t_{R}^{+}=\sup \{t: t>0 \text { and }|\Phi(s+i h)|<R \text { for } 0 \leqq s \leqq t\}, \\
& t_{R}^{-}=\inf \{t: t<0 \text { and }|\Phi(s+i h)|<R \text { for } t \leqq s \leqq 0\},
\end{aligned}
$$

and let $\Gamma_{h R}$ be the oriented Jordan arc whose parametrization is $\Phi(t+i h), t_{R}^{-} \leqq t$ $\leqq t_{R}^{+}$. The endpoints of $\Gamma_{h R}$ divide the circle $|z|=R$ into two arcs. The contour $G_{h R}^{1}$ is the Jordan curve which consists of $\Gamma_{h R}$ and an arc of the circle $|z|=R$ (oriented counterclockwise) and whose winding number at the point $z=0$ is +1 . The contour $G_{h R}^{0}$ is the Jordan curve which consists of $\Gamma_{h R}$ and an arc of the circle $|z|=R$ (oriented clockwise) and whose winding number at the point $z=0$ is 0 .

LEMMA 6. If $f, h, R$, and $F$ are as above, then for $z \in F$

$$
f(z)=\frac{1}{2 \pi i} \int_{G_{h R}^{0}} \frac{f(\zeta)}{\zeta-z} d \zeta .
$$

The lemma follows from the Cauchy integral formula. Indeed, the function $f$ is analytic on and inside $G_{h R}^{1}$, on and outside $|z|=R$, and $f(\infty)=0$. Therefore

$$
f(z)=\frac{1}{2 \pi i} \int_{G_{h R}^{1}} \frac{f(\zeta)}{\zeta-z} d \zeta \text { and } \frac{1}{2 \pi i} \int_{|\zeta|=R} \frac{f(\zeta)}{\zeta-z} d \zeta=0
$$

for $z \in F$. If the second integral is interpreted as a contour integral around the circle oriented clockwise, then we can add these two expressions to obtain (16).

Returning to the proof of the theorem, we make use of the Hermite formula $[7$, p. 50] to obtain

$$
f(z)-L_{N}[f ; z]=\frac{1}{2 \pi i} \int_{G_{h R}^{0}} \frac{p_{N}(z)}{p_{N}(\zeta)} \frac{f(\zeta)}{\zeta-z} d \zeta, \quad N=n_{j}
$$


for $z \in F$. If we apply Lemma 4 , then we can select $\lambda>1$ and $n_{0}$ such that $\left|p_{N}(z)\right| \leqq 2$ for $N=\left[n^{\lambda}\right], n>n_{0}$, and $z \in F$. Also, if we apply Lemma 5 with $G_{h R}^{0}$ as the compact set $E \subset \Omega^{*}$, then with the same constant $\lambda$ as above we have $\left|p_{N}(z)\right| \geqq M e^{2 \pi n h}$ for $z \in G_{h R}^{0}, N=\left[n^{\lambda}\right], n>n_{0}$. Combining these two estimates we conclude

$$
\sup _{z \in F}\left|f(z)-L_{N}[f ; z]\right| \leqq M_{1} e^{-2 \pi n h},
$$

$N=\left[n^{\lambda}\right], n>n_{0}$, where $M_{1}$ depends on $\Gamma, F$ and $f$ but not on $n$. The proof of the theorem is complete.

If instead of a function $f$ analytic on $\Omega \cup \Gamma$ we have a function $g$ analytic in a neighborhood of $\Gamma$ (including $\infty$ ), $g(\infty)=0$, then the Lagrange interpolating polynomials can again be formed. Using essentially the proof of Theorem 1, we can establish a convergence theorem in this case as well. Here the polynomials do not converge to $g$ in $\Omega$, indeed $g$ need not even be defined there, but to the Cauchy integral of $g$ over $\Gamma$. The necessary observation to supplement the proof of Theorem 1 is that for sufficiently large $R$ and sufficiently small $h>0$

$$
\int_{G_{h R}^{0}} \frac{g(\zeta)}{\zeta-z} d \zeta=\int_{\Gamma} \frac{g(\zeta)}{\zeta-z} d \zeta, \quad z \in \Omega
$$

THEOREM 2. Let $\Omega$ be an admissible region with boundary $\Gamma$. Then there is a family $\left\{S_{N}\right\}$ of sets of points on $\Gamma, S_{N}=\left\{z_{N k}\right\}_{k=1}^{N}, N=n_{1}, n_{2}, \ldots, n_{j} \rightarrow \infty$, such that if $g$ is any function analytic on $\Gamma$ (including $\infty$ ), then the sequence $L_{N}[g ; \cdot]$ of polynomials of respective degrees $N-1$ which interpolate to $g$ on $S_{N}$ satisfies

$$
\lim _{N \rightarrow \infty} L_{N}[g ; z]=\frac{1}{2 \pi i} \int_{\Gamma} \frac{g(\zeta)}{\zeta-z} d \zeta, \quad z \in \Omega,
$$

and the convergence is uniform on compact subsets of $\Omega$.

6. Remarks. A. It is reasonable to ask whether the admissibility conditions imposed on $\Omega$ in Theorem 1 are in any sense necessary for the conclusion. In this direction the following example indicates that some condition on the size of $\Omega$ at $\infty$ is necessary. Suppose that $\Omega$ is an unbounded region containing a half plane, which for convenience we assume to be the half plane $\operatorname{Re} z>0$, and that the conclusion of Theorem 1 holds for $\Omega$. That is, there is a family $\left\{S_{N}\right\}$ of sets of points on $\Gamma$ for which (15) is valid for any function $f$ analytic on $\Omega \cup \Gamma$. Let $x_{0}$ be real and contained in $\Omega^{*}$, set $f(z)=\left(z-x_{0}\right)^{-1}$, and let $F$ be any compact set in $\left\{\operatorname{Re} z \geqq\left|x_{0}\right|\right.$, $\operatorname{Im} z=0\}$. If we define $G_{h R}^{0}$ as in $\S 5$, which is possible independently of any smoothness assumptions on $\Gamma$, then we again have the expression (17). However, with the specific function $f$ considered here the right-hand side can be evaluated explicitly and we have

$$
f(z)-L_{N}[f ; z]=p_{N}(z) / p_{N}\left(x_{0}\right)\left(z-x_{0}\right), \quad z \in F .
$$

Thus, if (15) is to hold, then

$$
\lim _{N \rightarrow \infty}\left\{\sup _{z \in F}\left|\frac{p_{N}(z)}{p_{N}\left(x_{0}\right)}\right|\right\}=\lim _{N \rightarrow \infty}\left\{\sup _{z \in F} \prod_{k=1}^{N}\left|\frac{z-z_{N k}}{x_{0}-z_{N k}}\right|\right\}=0 .
$$


But the last product is a product of $N$ factors each of which is no smaller than 1 . Thus (18) is impossible and Theorem 1 is false for such a region.

In [2] J. M. Elkins proved the existence of families $\left\{S_{n}\right\}$ for which the associated polynomials $p_{n}$ satisfy $\lim _{n \rightarrow \infty} p_{n}(z)=1, z \in \Omega$, for quite general unbounded regions $\Omega$. The condition of [2] analogous to the admissibility condition of this paper is that $\Omega$ be contained in a half plane and not contain a half plane. The methods of that paper involve conformal mappings of a sequence of bounded regions and are nonconstructive.

B. Let $\Omega$ be a bounded Jordan region with boundary $\Gamma$. If $a \in \Gamma$ we say that $(\Omega, a)$ is an admissible pair if the region $\Omega^{\prime}=\{\mathscr{Z}: \mathscr{Z}=1 /(z-a), z \in \Omega\}$ is an admissible region in the $\mathscr{Z}$-plane in the sense of the Definition of $\S 2$. In this situation Theorem 1 can be restated in the following form.

THEOREM 3. Let $\Omega$ be a bounded Jordan region and $(\Omega, a)$ an admissible pair. Then there is a family $\left\{S_{N}\right\}$ of sets of points on $\Gamma \mid a$, each $S_{N}$ containing $N$ distinct points, with the following property. If $f$ is any function analytic in $\Omega \cup \Gamma$, then the sequence $R_{N}[f ; \cdot]$ of rational functions of respective degrees $N-1$ with their only pole at $z=a$ which interpolate to $f$ on $S_{N}$ satisfies

$$
\lim _{N \rightarrow \infty} R_{N}[f ; z]=f(z), \quad z \in \Omega,
$$

and the convergence is uniform on compact subsets of $\Omega$.

\section{REFERENCES}

1. J. H. Curtiss, Riemann sums and the fundamental polynomials of Lagrange interpolation, Duke Math. J. 8 (1941), 525-532. MR 3, 115.

2. J. M. Elkins, Approximation by polynomials with restricted zeros, J. Math. Anal. Appl. 25 (1969), 321-336. MR 38 \#2308.

3. L. Fejẻr, Interpolation und konforme Abbildung, Gött. Nachr. (1918), 319-331.

4. M. Thompson, Approximation by polynomials whose zeros lie on a curve, Duke Math. J. 31 (1964), 255-265. MR 28 \#4125.

5. J. L. Walsh, Note on polynomial interpolation to analytic functions, Proc. Nat. Acad. Sci. U.S.A. 19 (1933), 959-963.

6. - Approximation by polynomials in the complex domain, Mémorial des Sciences Mathématiques, Fasc. 73, Gauthier-Villars, Paris, 1935.

7. - Interpolation and approximation by rational functions in the complex domain, 3rd ed., Amer. Math. Soc. Colloq. Publ., vol. 20, Amer. Math. Soc., Providence, R. I., 1960. MR 36 \#1672a.

8. S. Warschawski, Uber das Randverhalten der Ableitung der Abbildungsfunktion bei konformer Abbildung, Math. Z. 35 (1932), 321-456.

9. —_ Z Zur Randverzerrung bei konformer Abbildung, Compositio Math. 1 (1935), 314-343.

Department of Mathematics, Indiana University, Bloomington, Indiana 47401

Department of Mathematics, University of Maryland, College Park, Maryland 